\title{
FEATURE A holistic strategy for adaptive land management
}

Jeffrey E. Herrick, Michael C. Duniway, David A. Pyke, Brandon T. Bestelmeyer,

Skye A. Wills, Joel R. Brown, Jason W. Karl, and Kris M. Havstad

A daptive management is widely applied to natural resources management (Holling 1973; Walters and Holling 1990). Adaptive management can be generally defined as an iterative decision-making process that incorporates formulation of management objectives, actions designed to address these objectives, monitoring of results, and repeated adaptation of management until desired results are achieved (Brown and MacLeod 1996; Savory and Butterfield 1999). However, adaptive management is often criticized because very few projects ever complete more than one cycle, resulting in little adaptation and little knowledge gain (Lee 1999; Walters 2007). One significant criticism is that adaptive management is often used as a justification for undertaking actions with uncertain outcomes or as a surrogate for the development of specific, measurable indicators and monitoring programs (Lee 1999; Ruhl 2007).

In this paper, we argue for a more holistic and systematic approach to adaptive management. We define holistic adaptive land management (HALM) as a refinement of adaptive management that requires (1) a process-based understanding of ecosystem dynamics and ecological mechanisms, (2) a willingness and ability to identify and consider all possible management alternatives, (3) rigorous monitoring of management effects, and (4) constant adaptation of management based on

Jeffrey E. Herrick is research soil scientist, Brandon T. Bestelmeyer and Jason W. Karl are research ecologists, and Kris M. Havstad is supervisory scientist at the Jornada Experimental Range, USDA Agricultural Research Service, Las Cruces, New Mexico. Michael C. Duniway is research ecologist at the Southwest Biological Science Center, US Geological Survey, Moab, Utah. David A. Pyke is research ecologist at the Forest and Rangeland Ecosystem Science Center, US Geological Survey, Corvallis, Oregon. Skye A. Wills is soil scientist at the National Soil Survey Center, USDA Natural Resources Conservation Service, Lincoln, Nebraska. Joel R. Brown is soil scientist at the Jornada Experimental Range, USDA Natural Resources Conservation Service, Las Cruces, New Mexico. monitoring data and associated observations. Thus, HALM requires both a model that accurately represents system dynamics (including drivers) and a monitoring system that includes indicators of both drivers and response variables, ensuring that monitoring data can be used to guide both proactive and reactive management.

While the scientific community has actively participated in the development of many variations of new tools that can facilitate HALM and often provides training in the use of specific protocols (Han et al. 2010), it has provided relatively little guidance on how to integrate and apply these tools to decision making. As a result, these tools are often underutilized and misapplied, sometimes leading to poorly informed decisions that result in undesirable outcomes. Use of monitoring protocols that are not comparable and a lack of systems for integrating and sharing comparable monitoring data further limit the usefulness of data that are collected. Finally, funding for natural resource management is often limited and, with national and global economic crises, is increasingly in flux. HALM can be used to more effectively target limited financial and personnel resources where they are likely to have the greatest impact. Its positive impact on budgets can be further increased through the adoption of common assessment and monitoring methods that generate data relevant to multiple management objectives (Toevs et al. 2011)

The objectives of this paper are to (1) briefly review the current status of assessment and monitoring tools and the application of synthetic conceptual models of ecosystem dynamics (state and transition models [STMs]) and identify current limitations relative to their application to HALM; and (2) present a strategy for implementing HALM on public and private rangeland. We conclude with an example of how the US Bureau of Land Management (BLM) has selected a set of core measurements and indicators in order to increase the flexible integration of monitoring into management across multiple objectives and spatial scales and jurisdictions and a brief discussion of future challenges.

\section{EXISTING TOOLS AND THEIR STRENGTHS AND LIMITATIONS}

Assessment. Most early rangeland assessments focused on forage production. Concerns about increased soil erosion and sedimentation and their effects on other ecosystem services increased in the early 20th century (Wooton 1908). By the 1970s, the US Forest Service, BLM, and Soil Conservation Service had all adopted indicators of runoff and soil erosion. However, vegetation indices continued to dominate through the early 1990s (Pyke and Herrick 2003). Assessment protocols in other countries continue to be strongly focused on plant community composition and forage production (Herrick et al. 2006a).

The concepts of soil quality, soil health (National Research Council 1993), and rangeland health (National Research Council 1994) were all developed in the latter part of the 20th century. These concepts were based on a more holistic, integrated understanding of the relationship between land management and ecosystem function.

During the past decade, a number of assessment protocols have been developed that address rangeland health by integrating, to varying degrees, soil and vegetation indicators of ecological processes (Pellant et al. 1999, 2005). Other protocols that were primarily designed for monitoring can also be used to make one-time assessments (Tongway and Hindley 2004; Milton and Dean 1996; Herrick et al. 2005), provided that suitable reference data are available.

Monitoring. The evolution of monitoring protocols has paralleled the development of new assessment tools. For example, new protocols are available that integrate soil and vegetation indicators, allowing changes in soil stability and hydrologic function to be routinely evaluated. These new protocols also indirectly 
reflect changes in energy flow and nutrient cycling by considering indicators such as litter amount and distribution.

The cost-effective application of both new and existing monitoring protocols to management is limited by a number of factors, including data quality and storage, lack of analysis and interpretation, redundant data collection by different organizations, inconsistent application of methods within organizations, inappropriate site selection and spatial extrapolation (e.g., representative vs. probabilistic sampling), and ineffective integration of ground- and remote-sensing approaches. Data quality and management can be enhanced through training and the adoption of standardized methods and electronic data collection tools. Redundant data collection can be reduced by selecting standard measurements that generate multiple indicators (see section An Example of Core Measurements and Indicators for LongTerm Monitoring).

Monitoring site selection can be improved through identification of ecological sites with state and transition models (discussed below), geographic information systems (GIS), and remote sensing-based stratification, including some of the new object-based classification systems (Karl and Maurer 2009). These tools used together with the type of expert knowledge used to select "key areas" in more traditional livestock monitoring programs can be used to increase the quality of spatial extrapolation. In addition, remote sensing indicators can complement and even replace ground-based indicators where spatially and temporally consistent relationships can be established (Booth and Tueller 2003; Hunt et al. 2003) and can even be used for retrospective monitoring when historic field-based data are unavailable (Washington-Allen et al. 2006; Malmstrom et al. 2009). High-resolution aerial photography, in particular, has the potential to revolutionize monitoring of composition and spatial patterns of the top canopy layer (Booth and Tueller 2003; Booth et al. 2006; Duniway et al. 2012).

One of the most significant and persistent limitations to the effective application of monitoring data to HALM is that the relationship between indicators and man- agement actions is often undefined or erroneous. As a result, monitoring data are often regarded as irrelevant to management decisions, or the data are misinterpreted. appropriate management actions or in the application of unnecessary actions. At best, this results in the misallocation of management resources; at worst, it can increase degradation or unnecessarily limit land use. These failures can often be avoided by clearly defining monitoring objectives, an often mentioned requirement for any monitoring program, and through research that more clearly establishes the relationship between indicators and the ecosystem properties and processes that the indicators are intended to represent.

It is crucial to clearly recognize and distinguish among three types of monitoring indicators: driver, short-term response, and long-term response (table 1). Indicators of drivers are used to quantify the type, timing, frequency, and intensity of disturbances (positive, neutral, and negative). This information is used to help explain changes in short- and long-term indicators. It is also often used to adjust management. The driver indicators should not be used as surrogates for response indicators except where a consistent relationship has been established. Examples of common indica-

\section{Table 1}

Questions addressed by short-term vs. long-term monitoring.

\begin{tabular}{ll}
\hline Questions addressed & Sample indicators \\
\hline Short-term monitoring & \\
\hline Is management plan being followed? & $\begin{array}{l}\text { Number of livestock or off-highway vehicle users } \\
\text { per unit time and area }\end{array}$ \\
Is management plan having desired & Stubble height \\
short-term effect? & Residual dry matter \\
\hline Long-term monitoring & Number of vehicle tracks per unit area \\
\hline What are the trends in critical ecosystem & Perennial grass basal cover \\
properties (which are related to processes)? & Invasive species cover \\
& Soil aggregate stability \\
What are the trends in critical ecosystem & Soil carbon content \\
processes? & Runoff and erosion rates \\
\hline
\end{tabular}
unit area. long-term indicators. Either one can result in failure to take

tors of drivers include animal unit months and off-highway vehicle miles driven per

Short-term response indicators are used to quantify the direct effects of drivers on soil, vegetation, or animals. This information is used to help explain changes in long-term indicators. It is also often used to adjust management. Short-term indicators should not be used as surrogates for long-term indicators except where a consistent relationship has been established. Examples of common short-term indicators include utilization and stubble height. The Stubble Height Review Team (2006) referred to these as trigger or endpoint indicators in contrast to

Long-term response indicators are used to quantify changes in ecological properties and processes. This information is used to adjust management and to enhance our understanding of the effects of different drivers on these properties and processes. Examples of long-term indicators include plant functional group composition, soil organic matter, and soil aggregate stability. Some indicators can serve as both shortand long-term indicators. For example, the proportion of the soil surface in semiarid grasslands covered by intercanopy gaps longer than 1 to $2 \mathrm{~m}$ (3.3 to $6.6 \mathrm{ft})$ can be 
used as a short-term indicator to trigger rotation of livestock where minimizing wind erosion is a management objective. The same indicator compared over a number of years reflects long-term changes in the spatial structure of the vegetation. In the strategy described below, we identify which type of indicator is appropriate for each stage of the decision process.

State and Transition Models. State and transition models are synthetic, conceptual models that describe soil and vegetation dynamics (Bestelmeyer et al. 2003, 2004, 2009; Briske et al. 2005, 2006, 2008; Stringham et al. 2003; Groffman et al. 2006; Herrick et al. 2006b). Hundreds of unique models have been developed for individual groups of soils, or ecological sites that are with similar potential production and response to management. There have been several articles discussing their broader relevance to other types of land use (Brown et al. 1999; Herrick et al. 2002). These models can be used to identify which parts of the landscape are most likely to change in response to different types of management and to identify indicators that are most likely to be sensitive to these changes. They can also be used to identify areas that are likely to require active intervention to support development of a desired plant community (figure 1). Good state and transition models either implicitly or explicitly identify both drivers and short-term response indicators in the description of transitions and pathways. Long-term indicators are generally part of the community and state descriptions.

In the strategy described below, state and transition models are used as an assessment tool, to help determine whether or not management intervention is likely to be cost-effective, to help identify specific management actions, and to identify indicators to evaluate the effects of those actions.

\section{A STRATEGY FOR IMPLEMENTING HOLISTIC ADAPTIVE LAND MANAGEMENT}

Overview. The objective of this strategy is to help managers integrate and more cost-effectively apply assessment and monitoring tools and state and transition models into their management decisions,

\section{Figure 1}

Application of a state and transition model to restoration ecology. Transitions among communities within an individual state generally require few external inputs, while transitions among communities in different states require active intervention.

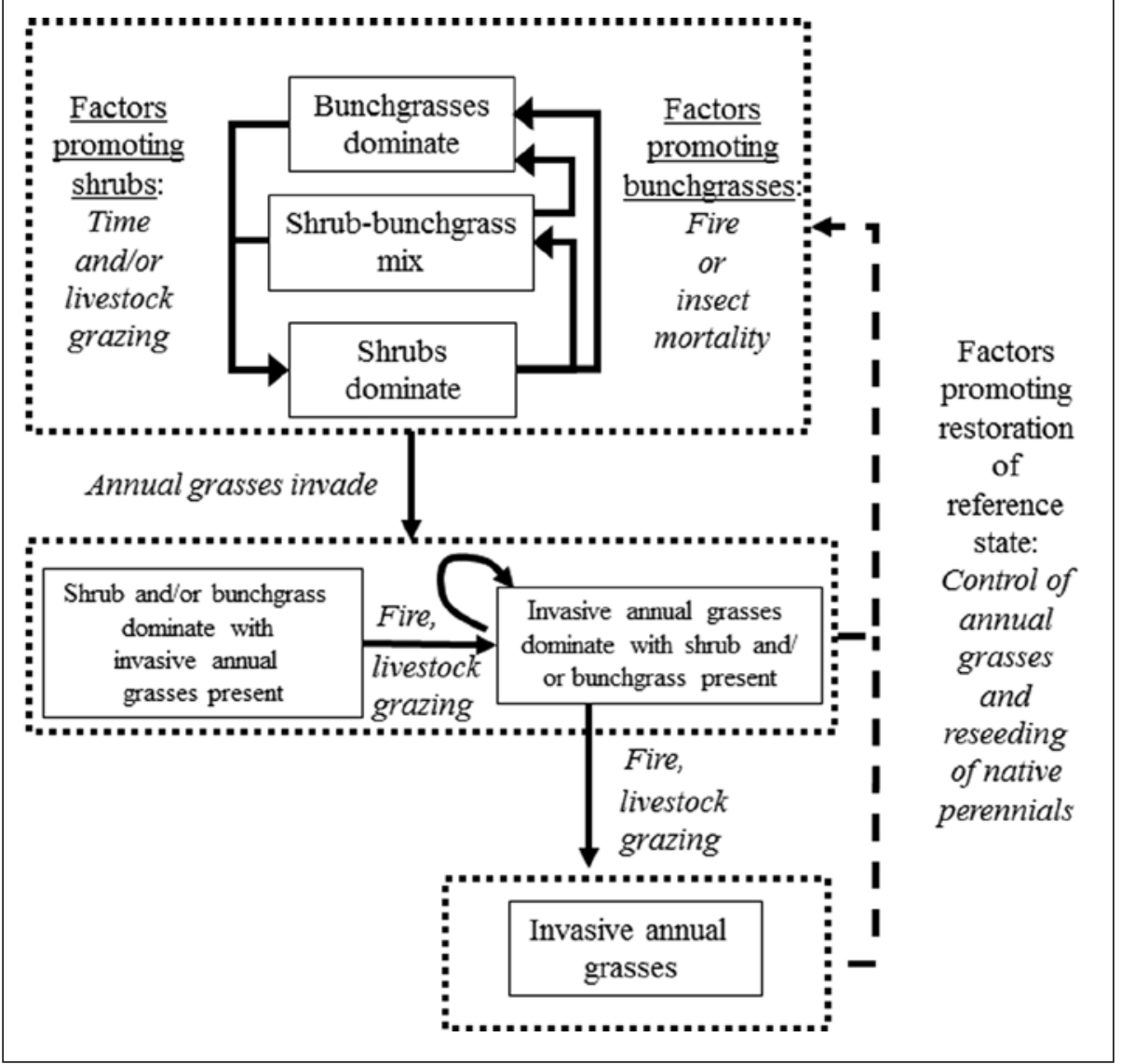

while avoiding many of the pitfalls associated with the individual application of these tools. We believe it is essential that these tools be used not as ends in themselves, but as elements of a HALM process where the tools are applied flexibly and iteratively. By clearly defining how each of the tools can be used at each stage of decision making, the strategy helps apply the tools most effectively, while helping to understand where research is needed to improve or replace the tools.

The strategy is organized around a series of questions, where the answer to each question leads to a decision and another question. It treats management as a series of hypothesis tests about the status of the land, the factors controlling its dynamics, and the land's response to specific management actions. By addressing each question in a holistic context that includes all potentially interacting factors, it allows the land manager to reduce the risk of misinterpretation of management impacts, for example by confusing the impacts of a change in forage quantity with forage quality or off-road vehicle impacts with overgrazing (see figures 2 and 3 ). Concerns about the environmental effects of off-road vehicle tracks are increasing in the United States and globally (Keshkamat et al. 2012), but the relative importance of these impacts has not been established for most ecosystems.

The strategy consists of two primary components: problem evaluation and management. Together, these form a decision-support system. In the evaluation component, the primary objective is to determine where management actions are required. In the management component, the objectives are to determine what management actions to implement and to adapt management over time. Both components include monitoring. The strategy is intentionally flexible and can be applied to a 
single resource concern or expanded to integrate multiple resource concerns. It is based on the assumption that a fundamental objective of nearly every landowner and of virtually all natural resource policies is to sustain the land's potential to support the delivery of ecosystem services. At the same time, the strategy can be used to guide management of particular types of uses. Adoption of a core suite of measurements and indicators for long-term monitoring helps ensure that the data collected can be used to address both current and future management questions.

Each component is presented as a decision tree. The basic structure is generic and should be reviewed, revised, and expanded or reduced to meet local requirements. The key point is that each fork in the tree is a hypothesis that is tested by a specific type of information. In the examples below, we have limited ourselves to dichotomous choices. In many cases, it may be more appropriate to incorporate multiple alternative hypotheses. This approach is not new-nearly every management action is a test of an often unstated hypothesis. Our objectives in presenting this strategy are to illustrate how assessment and monitoring tools can be effectively integrated into the decision process by landowners and other land managers and to promote adaptive management that is proactive and holistic rather than reactive (Brown and MacLeod 1996). Because the strategy is generic, it can be used with many of the conceptual models and assessment and monitoring protocols currently being applied to lands around the world. For simplicity, we have illustrated the strategy primarily with examples from state and transition models as used in the United States and the assessment and monitoring protocols described in Pellant et al. (2005) and Herrick et al. (2005).

Ultimately, this strategy is designed to increase our ability to promote science-based management. Science-based management is management based on knowledge that has been tested through experimentation where available, on hypotheses where this knowledge is unavailable and, most commonly, on a combination of knowledge and hypotheses that are tested and supported by

\section{Figure 2}

Sample decision tree for the problem evaluation component of a decision support system for off-highway vehicle (OHV) impact management.

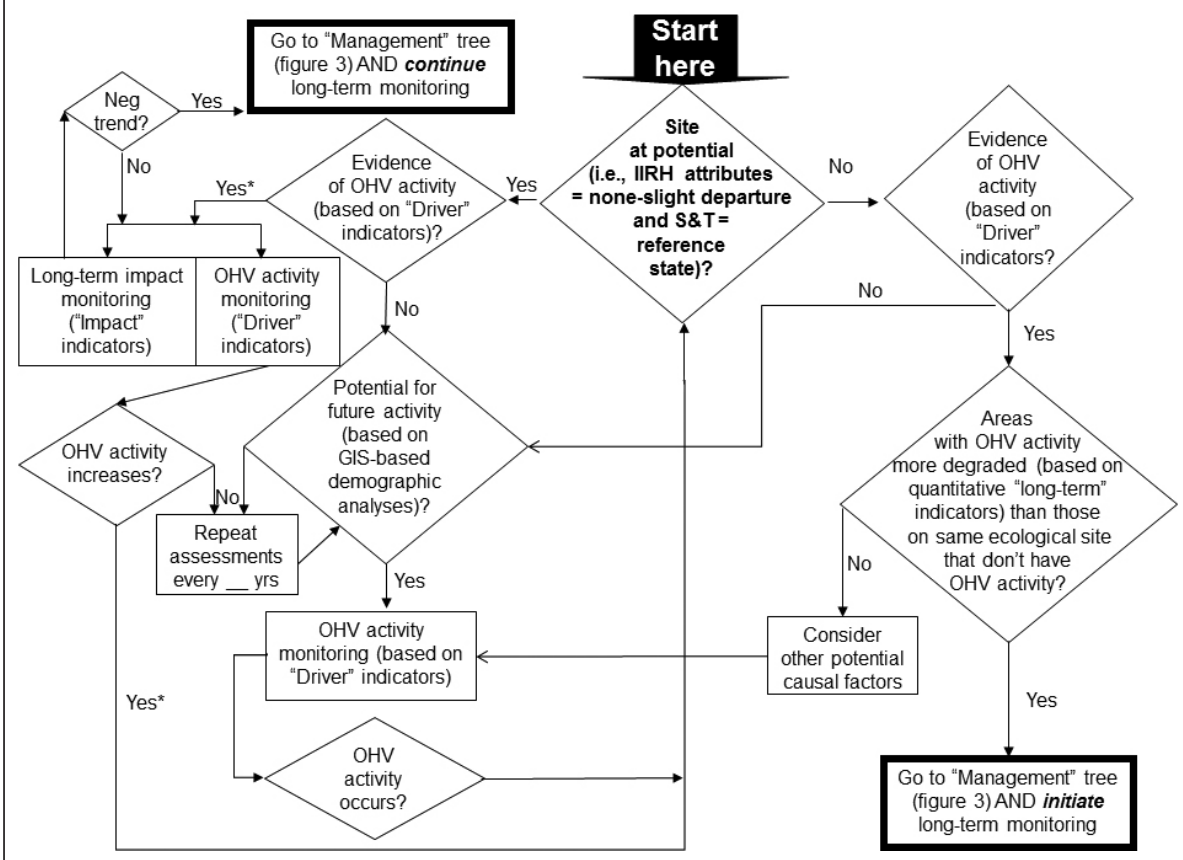

Notes: IIRH = "Interpreting Indicators of Rangeland Health" (Pellant et al. 2005). S\&T = state and transition model.

* Management prevention response (e.g., warning signs) may also be required.

\section{Figure 3}

Sample decision tree for the management component of a decision support system for off-highway vehicle (OHV) impact management. This is applied after an assessment (figure 2) indicates that degradation has occurred.

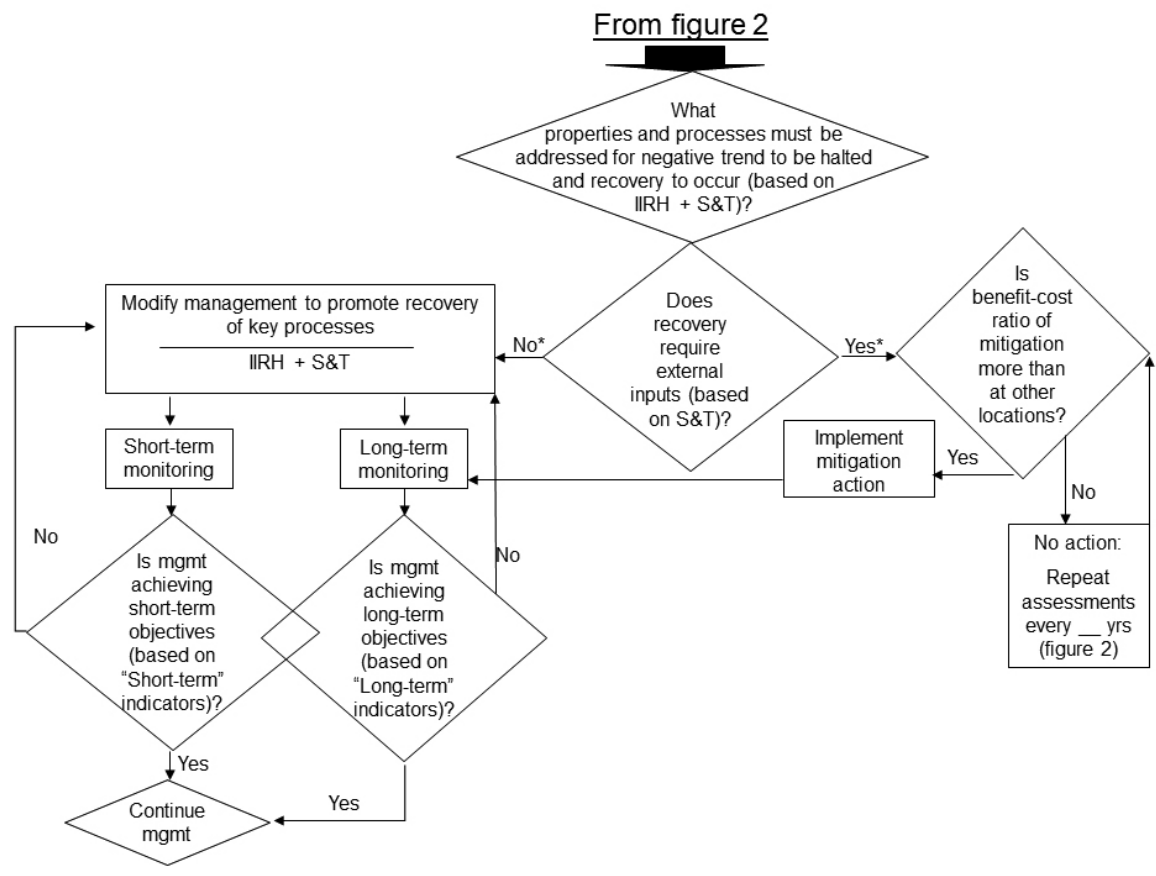

Notes: IIRH = “Interpreting Indicators of Rangeland Health" (Pellant et al. 2005). S\&T = state and transition model.

* Management prevention response (e.g., warning signs) may also be required. 
assessment and monitoring data collected or assembled as part of the decision process. Knowledge that has been tested through experimentation includes both the results of published experiments and local or traditional knowledge derived from careful monitoring and observations.

By acknowledging that many of our decisions are, in fact, hypotheses, we are more likely to assemble the information to test them. This information is then used to support the resulting decisions.

Problem Evaluation Component. Qualitative assessments, optionally and optimally supplemented by quantitative data, are used to identify the ecological status of areas and to help managers understand which ecological processes have been compromised. State and transition models are used together with these assessments to further focus attention on areas that are at risk of undergoing a threshold transition. A threshold transition is defined as one that, over time, is increasingly unlikely (Briske et al. 2006) to be reversed without significant external inputs. These models are also used to improve economic analyses, helping managers define what is possible (all plant communities included in the model) and what is realistic (generally plant communities in the current state [figure 1 and table 2]). Finally, they are used together with the qualitative assessments to determine what type of monitoring data are required to inform future management decisions and where monitoring plots should be located. In the example below, we have attempted to identify which type of indicator is appropriate for each stage of the decision process (drivers, short-term response and long-term response).

Figure 2 shows an example of a decision tree for the evaluation component. This example is designed to determine if an area is degraded relative to its potential and whether or not the degradation is associated with off-highway vehicle (OHV) activity. The sequence begins with a general question or hypothesis about the current status of the land relative to its potential. This general hypothesis includes five specific subhypotheses. The first three indicate that the land is functioning at or near its potential (defined as "none-slight

\section{Table 2}

Relative costs of converting to a desired community from the current community for a simple three-state system (figure 1).

\begin{tabular}{llll}
\hline Desired community & \multicolumn{2}{l}{ Current community } & \\
\cline { 2 - 4 } & Reference state & Intermediate state & Degraded state \\
\hline Reference state & Low & High & Very high \\
Intermediate state & Low* & Low & High \\
Degraded state & Low* & Low* & Low \\
\hline * These conversions incur an opportunity cost (loss of future long-term options or increased cost of \\
reconversion) and may incur immediate costs in lost ecosystem services.
\end{tabular}

departure" in figure 2, based on the nationally applied protocol described in Pellant et al. 2005) with respect to the three rangeland health attributes: soil and site stability, hydrologic function, and biotic integrity. These hypotheses are evaluated using the qualitative indicators described in "Interpreting Indicators of Rangeland Health" (IIRH) supported by quantitative measurements for key indicators (Pellant et al. 2005).

The other two hypotheses embedded in the decision tree (figure 2) are tested in the context of the ecological sitespecific STM: (1) the evaluation area is in a nondegraded state and (2) it is not approaching a threshold transition. State indicators generally focus on plant community composition and structure, but should be supplemented by process indicators (e.g., from the rangeland health protocol), particularly when evaluating the hypothesis that the area is at or near a threshold transition.

In this example, both branches of the tree lead to questions about OHV activity, but the hypotheses are different. In the nondegraded case (left side of figure 2 where the site is determined to be at its ecological potential), the hypothesis is simply that OHV activity is occurring. Simple indicators of presence (tracks or sightings) are sufficient. In the degraded case (right side of figure 2), causation must often be determined. Simple indicators of OHV presence are necessary but not sufficient to assign cause. An additional hypothesis (not shown) that areas with evidence of OHV activity are more degraded than similar areas (e.g., same ecological site) without evidence of activity must be tested using a combination of qualitative (IIRH) and quantitative soil and vegetation indicators (Duniway et al. 2010). Each of the two major branches ultimately leads to decisions about whether, what, and where to monitor. Where the potential exists for increased OHV activity, the driver (OHV activity) must be monitored using track counts or (ideally) electronic counters. Where a threshold level of degradation has been identified to trigger a management response, a combination of short-term and long-term impact monitoring is appropriate. In all cases, the monitoring data are used together with repeated assessments in the feedback loops shown in figure 2 .

An understanding of the relationships between individual assessment indicators and specific ecological processes can be used to both identify sensitive monitoring indicators and to focus management on those actions with the greatest potential impact. An example is illustrated in figure 4. This example also illustrates how existing data from similar ecological sites (figure 5) can be used to help refine hypotheses about indicator process relationships. Where ecological site-specific data do not exist, generic models can often be applied (Okin 2008).

Management Component. The results of the assessment component are used together with STMs to identify a range of management options. Benefit-cost and risk analyses are then used to select one or more options. The results of the assessment component are then applied together with state and transition models to select appropriate monitoring indicators. After the management action has been initiated, monitoring data and repeated assessments are used to modify management. 
Figure 3 shows an example of a decision tree for the management component for degradation associated with OHV activity. It begins by drawing on the previous assessments (qualitative, quantitative, and STM based) to identify the properties and processes that must be addressed for recovery to occur, which leads to the first hypothesis-recovery requires external inputs. This hypothesis is addressed using a combination of expert knowledge and the appropriate state and transition models. If no external inputs are required to restore or remediate a site, management changes are selected that address the limiting processes and properties. This step is deceptively simple, but in fact generates one or more new hypotheses that must be tested with appropriate short- and longterm indicators if management is to be adapted to continue to promote recovery (see arrows leading back to the "Modify management to promote recovery of key processes" box in figure 3). An example of how a process-based assessment of wind erosion thresholds is based on quantitative data is illustrated in figure 4.

For systems where external inputs are required, a benefit-cost analysis should be completed to determine whether applying inputs to this location is likely to generate more significant benefits to society than if they were applied to other locations. These analyses are often not initiated because they appear too complex and the results are too uncertain. In addition, the analyses should incorporate a landscape or regional approach that integrates potential restoration success based on current ecological state and the status of selected indicators (Meinke et al. 2009) with local, regional, or national priorities for optimizing particular ecosystem services. We believe that even a qualitative analysis that simply lists the potential benefits along with all of the costs can significantly increase the cost-effectiveness of management. In New Mexico alone, where the BLM applied restoration treatments to over 404685 ha $(1,000,000 \mathrm{ac})$ between 2006 and 2009, application of this type of analysis could result in significantly increased return on investment. Application of short- and long-term monitoring of the key properties and processes necessary for recovery

\section{Figure 4}

Example of how a qualitative assessment of a wind erosion threshold is used to define management objectives and identify quantitative monitoring indicators for wind-erodible soils. Data shown are threshold wind velocities at $2 \mathrm{~m}$ necessary to initiate movement of disturbed wind-erodible fine sand in Chihuahuan Desert grasslands ( $n=25$ observations). Each point represents a different intercanopy gap (Herrick et al. 2005). Gap diameter is measured parallel to wind.

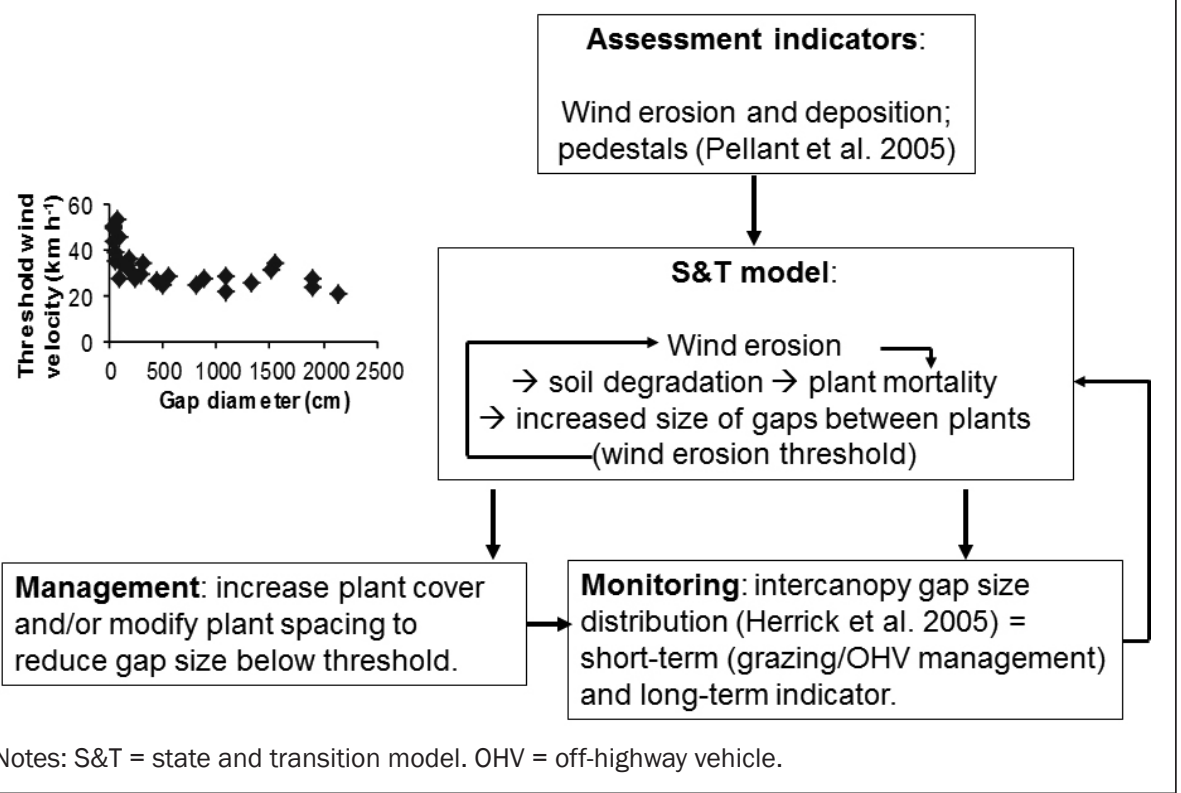

\section{Figure 5}

Illustration of how extensive, long-term regional monitoring data can be integrated with repeated assessments and qualitative observations to create an adaptive monitoring program that supports adaptive, hypothesis-based, management.

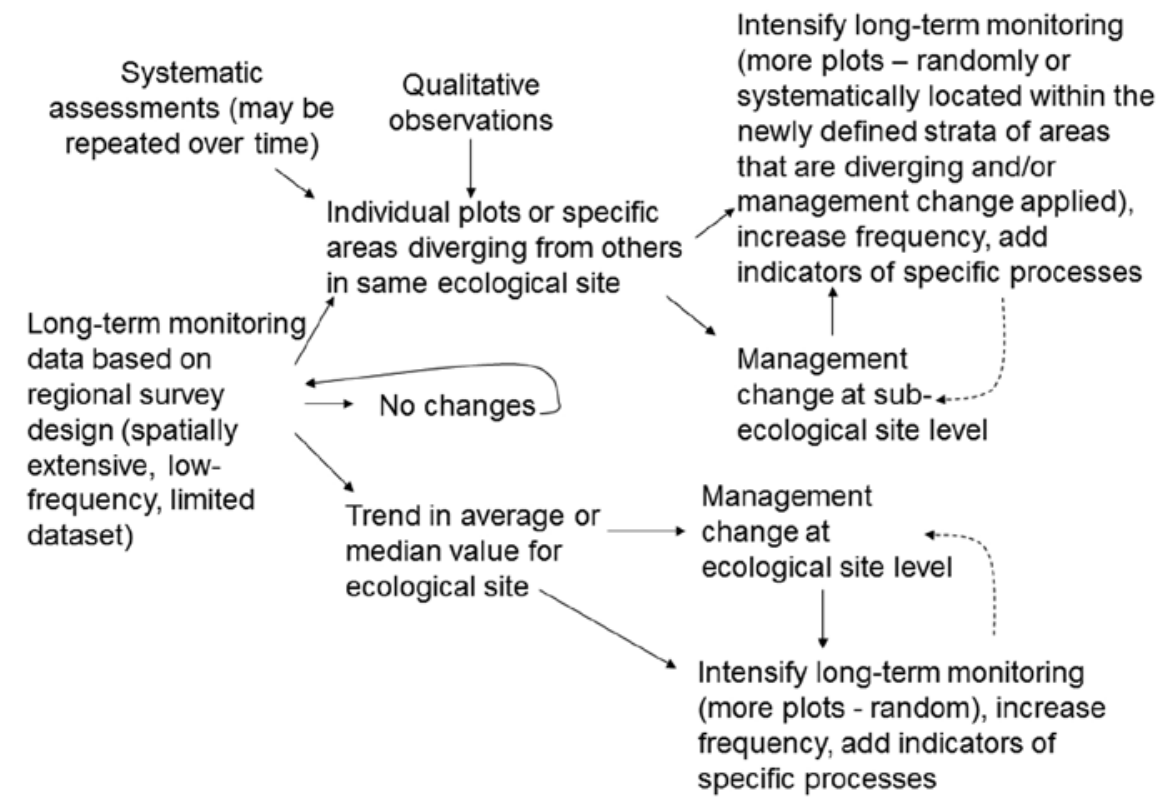

must be an integral part of the process if the objective is to achieve sustained recovery of these areas.

Core Measurements and Indicators for Long-Term Monitoring. A core set of measurements and indicators for longterm monitoring is an important part of a long-term management strategy, particularly where management actions must be coordinated or prioritized across different 
land management units and where multiple groups with different management and monitoring objectives are working within the same management unit. Both situations are characteristic of land management in many countries, including the United States where land tenure patterns are often reflected in a checkerboard pattern and wildlife, range, and recreation specialists within the same agency often employ unique and overlapping approaches to monitoring.

Most management questions can be addressed by a relatively small suite of core ecosystem properties that serve as indicators of a wide range of ecosystem processes on which most management objectives depend. The ability of different groups and disciplines to share the types of information needed to make the decisions identified in figures 2 and 3 at appropriate scales is hampered by inconsistent methods and indicators.

While the optimal methods and indicators vary, many methods can be used to generate multiple indicators, and often even suboptimal indicators can provide adequate interpretations. Where they do not, supplementary indicators should be added to a core monitoring program. The cost savings of adopting a consistent, core set of monitoring measurements can be significant by reducing redundant data collection. Future benefits may be even greater as research managers are able to use data collected for other objectives as baseline data to address new questions.

For example, bare ground is generally related to changes in susceptibility to erosion, biomass production, and wildlife habitat quality. By adopting a clear definition of bare ground and a method for measuring it, it would be possible to make landscape-scale interpretations about the status of multiple processes both within the BLM and across agency boundaries.

While adoption of a core set of measurements and indicators can increase the utility of monitoring data, it can also lead to the inadvertent omission of indicators necessary to address specific questions. Where completely different types of monitoring data are required in different areas, it is often possible to make inferences across larger regions by aggregating interpretations of the data rather than the data themselves.

\section{AN EXAMPLE OF CORE MEASUREMENTS AND INDICATORS FOR LONG-TERM MONITORING}

The United States Bureau of Land Management recognized the benefits of standardizing monitoring data collection and initiated a process in 2008 to identify a core set of measurements and long-term indicators that could be applied across rangeland, riparian, and forest lands, with the understanding that they would often need to be supplemented by additional measurements. This process was coordinated by an interdisciplinary team of BLM managers and facilitated by the USDA Agricultural Research Service and US Geological Survey scientists.

Indicators were selected through a fivestep participatory process involving both BLM and outside experts. Nearly 200 individuals contributed to this process during a three-month period from August to November 2008. A total of 16 criteria (table 3) were developed and systematically applied (Likert 1931; Trochim 2006) to a set of candidate indicators previously identified by the US Forest Service Forest Inventory and Analysis program, the USDA Natural Resources Conservation Service National Resources Inventory (NRCS NRI), the Heinz Center (State of the Nation's Ecosystems), and the Sustainable Rangelands Roundtable. Similar indicators from these sources were combined, and indicators that clearly did not meet a number of the criteria were eliminated, reducing the size of the candidate set to 18 indicators.

Following additional internal and external review, six core indicators were selected: bare ground, vegetation composition, nonnative invasive species, plant species of management concern, vegetation height, and proportion of the soil surface in large, intercanopy gaps (Toevs et al. 2011). These indicators are quantified using just three core field methods: line point intercept, maximum height at selected line point intercept points, and canopy gap (Herrick et al. 2005). Two contingent indicators were also selected: soil aggregate stability, for application where soils are potentially unstable, and soil toxins, where believed to be present. This set of indicators is now being implemented nationally through the BLM's Assessment, Inventory, and Monitoring Program. Because the core indicators are also applied nationally by the NRCS NRI and locally by the USDA NRCS, BLM's action effectively created a national standard for rangeland inventory and monitoring.

\section{FUTURE CHALLENGES}

There are three significant challenges that limit the application of the strategy described above: threshold determinations, ability to interpret changes in a landscape context, and education and communication. The strategy recommends testing the hypothesis that an area has crossed a threshold and is in a degraded state by using the best available qualitative and quantitative indicators. This is a critical step because areas that have crossed a threshold are difficult, if not impossible, to restore to the original state. Threshold determinations are also highly uncertain in most ecosystems. Threshold determinations are generally made based on a single point-in-time evaluation. Increased use must be made of long-term monitoring data from similar systems where a threshold transition has been confirmed. Where a high level of technical support is available, expert knowledge may be combined with state and transition models using Bayesian belief networks (Bashari et al. 2009) to improve the quality of hypotheses that threshold transitions have occurred.

The second challenge is that our ability to interpret changes in a landscape context is limited by our poor understanding of the complexity of landscape dynamics, by the ecological site basis of the approach itself, and by an inadequate understanding or documentation of ecological processes and mechanisms (Boyd and Svejcar 2009). Focusing evaluations on individual landscape units is similar to conducting forest health evaluations by focusing on herbivore pressure on individual trees without considering insect populations in the surrounding forest. The strategy described above must evolve to address landscapescale patterns and interactions. 
The third challenge is education. The science associated with this strategy is evolving rapidly, increasing numbers of landowners have little or no training in land management, and federal land managers are under increasing pressure to be more efficient in their management. Local knowledge that could be used to complement published data is rapidly being lost before it can be communicated. While this decision tree approach is an attempt to increase managers' ability to select the appropriate tool for each decision, it is worthless without an understanding of how to apply each of the tools. This requires that both scientists and managers devote increasing amounts of time to education and communication.

\section{SUMMARY AND CONCLUSIONS}

New assessment and monitoring tools, including STMs, provide managers with new opportunities to optimize and adapt management. However, a more systematic strategy is needed to effectively integrate and apply these tools. Holistic adaptive land management (HALM) consists of two primary components: problem evaluation and management. Both components include monitoring. Adoption of a core suite of measurements and indicators for long-term monitoring helps ensure that the data collected can be used to address both current and future management questions. Flexible decision trees are used to integrate and apply these tools to specific management objectives. HALM can be used to prioritize management and restoration efforts, design cost-effective assessment and monitoring programs, and implement adaptive management on public and private lands, with a focus on rangelands. BLM's recent adoption of core indicators and methods already applied by the USDA NRCS have created a national standard for rangeland inventory and monitoring, creating opportunities for landscape-scale conservation efforts across public and private rangelands.

\section{ACKNOWLEDGEMENTS}

We thank Craig Mackinnon, Gordon Toevs, and Kit Muller of the US Bureau of Land Management, Washington, DC; Sherm Karl of the US Bureau of Land Management, Denver, Colorado; and the rest of the US Bureau of Land Management team that contributed to the development of the core indicators. Angela Muhanga of New Mexico State University, Las Cruces, New Mexico assisted with the compilation of the surveys. We are grateful to Arlene Tugel of the USDA Natural Resources Conservation Service, Las Cruces, New Mexico; Dennis Thompson and Leonard Jolley of the USDA Natural Resources Conservation Service, Washington, DC; Pat Shaver of the USDA Natural Resources Conservation Service, Portland, Oregon; Mike Pellant of the US Bureau of Land Management, Boise, Idaho; Fee Busby of the Utah State University, Logan, Utah; Robert Unnasch of The Nature Conservancy, Boise, Idaho; Anton Imeson of the 3D Environmental Change, The Netherlands; Luca Montanarella of the European Commission Joint Research Center, Ispra, Italy; Michelle Wander of the University of Illinois, Urbana, Illinois; Shannon Horst of the Savory Institute, Boulder, Colorado; Craig Leggett of Leggett Consulting, Durango, Colorado; Corinna Riginos of Mpala Research Center, Nanyuki, Kenya; Jayne Belnap of the US Geological Survey, Moab, Utah, and many others for conversations that contributed to the development of this manuscript. Work associated with this manuscript was supported by the US Bureau of Land Management and by the USDA Agricultural Research Service and the USDA NRCS through the Conservation Effects Assessment Project. Any use of trade, product, or firm names is for descriptive purposes only and does not imply endorsement by the US Government..

\section{REFERENCES}

Bashari, H., C. Smith, and O.J.H. Bosch. 2009. Developing decision support tools for rangeland management by combining state and transition models and Bayesian belief networks. Agricultural Systems 99:23-3.

Bestelmeyer, B.T., J.E. Herrick, J.R. Brown, D.A. Trujillo, and K.M. Havstad. 2004. Land management in the American Southwest: A state and transition approach to ecosystem complexity. Environmental Management 34: 38-51.

Bestelmeyer, B.T., J.R. Brown, K.M. Havstad, R. Alexander, G. Chavez, and J.E. Herrick. 2003. Development and use of state and transition models for rangeland management. Journal of Range Management 56:114-126.

Bestelmeyer, B.T., A.J. Tugel, G.L. Peacock Jr., D.G. Robinett, P.L. Shaver, J.R. Brown, J.E. Herrick, H. Sanchez, K.M. Havstad. 2009. State-andtransition models for heterogeneous landscapes: A strategy for development and application. Rangeland Ecology and Management 62:1-15.
Booth, D.T. and P.T. Tueller. 2003. Rangeland monitoring using remote sensing. Arid Land Research and Management 17:455-467.

Booth, D.T., S.E. Cox, and R.D. Berryman. 2006. Precision measurements from very-large scale aerial digital imagery. Environmental Monitoring and Assessment 112:293-307.

Boyd, C.S., and T.J. Svejcar. 2009. Managing complex problems in rangeland ecosystems. Rangeland Ecology and Management 62:491-499.

Briske, D.D., S.D. Fuhlendorf, and F.E. Smeins. 2005. State-and-transition models, thresholds, and rangeland health: A synthesis of ecological concepts and perspectives. Rangeland Ecology and Management 58:1-10.

Briske, D.D., S.D. Fuhlendorf, and F.E. Smeins. 2006. A Unified framework for assessment and application of ecological thresholds. Rangeland Ecology and Management 59:225-236.

Briske, D.D., B.T. Bestelmeyer, T.K. Stringham, and P.L. Shaver. 2008. Recommendations for development of resilience-based state-and-transition models. Rangeland Ecology and Management 61:359-367.

Brown, J.R., and N.D. MacLeod. 1996. Integrating ecology into natural resource management policy. Environmental Management 20:289-296.

Brown, J.R., J.E. Herrick, and D. Price. 1999. Managing agroecosystems sustainably: The importance of ecological thresholds. Canadian Journal of Forest Research 29:1112-1119.

Duniway, M.C., J.E. Herrick, D.A. Pyke, and D.P. Toledo. 2010. Assessing Transportation Infrastructure Impacts on Rangelands: Test of a Standard Rangeland Assessment Protocol. Rangeland Ecology and Management 63:524-536.

Duniway, M., J. Karl, S. Schrader, N. Baquera, and J.E. Herrick. 2012. Rangeland and pasture monitoring: an approach to interpretation of high-resolution imagery focused on observer calibration for repeatability. Environmental Monitoring and Assessment 184:3789-3804.

Groffman, P.M., J.S. Baron, T. Blett, A.J. Gold, I. Goodman, L.H. Gunderson, B.M. Levinson, M.A. Palmer, H.W. Paerl, G.D. Peterson, N.L. Poff, D.W. Rejeski, J.F. Reynolds, M.G. Turner, K.C. Weathers, and J. Wiens. 2006. Ecological thresholds: The key to successful environmental management or an important concept with no practical application? Ecosystems 9:1-13.

Han, G., J.E. Herrick, B.T. Bestelmeyer, D.A. Pyke, P. Shaver, M. Hong, M. Pellant, F. Busby, and K.M. Havstad. 2010. Learning natural resource assessment protocols: Elements for success and 
lessons from an international workshop in Inner Mongolia, China. Rangelands 33: 2-9.

Herrick, J.E., J.R. Brown, A. Tugel, P.L. Shaver, and K.M. Havstad. 2002. Application of soil quality to monitoring and management: Paradigms from rangeland ecology. Agronomy Journal 94:3-11.

Herrick, J.E., E. Garcia-Moya, W. Willms, B Bestelmeyer, P. Sundt, and W. Barnes. 2006a. Arid and semi arid rangeland monitoring in North America. Science et Changements Planétaires Sécheresse 17:235-241.

Herrick, J.E., B.T. Bestelmeyer, S. Archer, A. Tugel, and J.R. Brown. 2006b. An integrated framework for science-based arid land management. Journal of Arid Environments 65:319-335.

Herrick, J.E., J.W. Van Zee, K.M. Havstad, L.M. Burkett, and W.G. Whitford. 2005. Monitoring Manual for Grassland, Shrubland and Savanna Ecosystems. Las Cruces, NM: Jornada Experimental Range, USDA Agricultural Research Service. Distributed by University of Arizona Press. http://jornada.nmsu.edu/ monit-assess.

Holling, C.S. 1973. Resilience and stability of ecological systems. Annual Review of Ecology and Systematics 4:1-23.

Hunt, E.R. Jr., Everitt, J.H., Ritchie, J.C., Moran, M.S., Booth, T.D., Anderson, G.L., Clark, P.E., and M.S. Seyfried. 2003. Applications and research using remote sensing for rangeland management. Photogrammetric Engineering and Remote Sensing 69:675-693.

Karl, J.W., and B.A. Maurer. 2009. Multivariate correlations between imagery and field measurements across scales: Comparing pixel aggregation and image segmentation. Landscape Ecology, doi:10.1007/s10980-009-9439-4.

Keshkamat, S.S., N-E. Tsendbazar, M.H.P. Zuidgeest, A. van derVeen, and J. de Leeuw. 2012. The environmental impact of not having paved roads in arid regions: An example from Mongolia. Ambio 41:202-205.

Lee, K.N. 1999. Appraising adaptive management. Ecology and Society 3:3.

Likert, R. 1931. A technique for the measurement of attitudes. Archives of Psychology. New York: Columbia University Press.

Malmstrom,C.M.,Butterfield,H.S.,Barber,C.,Dieter, B., Harrison, R., Qi, J., Raiño, D., Schrotenboer, A., Stone, S., Stoner, C.J., and J. Wirka. 2009. Using remote sensing to evaluate the influence of grassland restoration activities on ecosystem forage provisioning services. Restoration Ecology 17:526-538.
Meinke, C.W., S.T. Knick and D.A. Pyke. 2009. A Spatial model to prioritize sagebrush landscapes in the intermountain west (United States) for restoration. Restoration Ecology 17:652-659.

Milton, S.J., and W.R.J. Dean. 1996. Karoo Veld: Ecology and Management. Pretoria, South Africa: ARC-Range and Forage Institute.

National Research Council. 1993. Soil and Water Quality: An Agenda for Agriculture. Washington, DC: National Academy Press.

National Research Council. 1994. Rangeland health: new methods to classify, inventory, and monitor rangelands. Washington, D C: National Academy Press.

Okin, G.S. 2008. A new model of wind erosion in the presence of vegetation. Journal of Geophysical Research-Earth Surface, 113, F02S10, doi:10.1029/2007JF000758.

Pellant, M., P. Shaver, D. Pyke, and J.E. Herrick. 1999. Interpreting Indicators of Rangeland Health, Version 3. Interagency Technical Reference 17346. Denver, CO: Bureau of Land Management.

Pellant, M., P. Shaver, D. Pyke, and J.E. Herrick. 2005. Interpreting Indicators of Rangeland Health, Version 4. Interagency Technical Reference 1734 6. Denver, CO: Bureau of Land Management. http://jornada.nmsu.edu/monit-assess.

Pyke, D.A., and J.E. Herrick. 2003. Transitions in rangeland evaluations. Rangelands 25(6):22-30.

Ruhl,J.B. 2007. Regulation by adaptive management - is it possible? Minnesota Journal of Law, Science \& Technology 7:21-57.

Savory, A., and J. Butterfield. 1999. Holistic Management: A New Framework for Decisionmaking. 2nd ed.Tucson, AZ: Island Press. Stringham, T.K., W.C. Krueger, and P.L. Shaver. 2003. State and transition modeling: An ecological process approach. Journal of Range Management 56(2):106-113.

Stubble Height Review Team. 2006. Using stubble height to monitor riparian vegetation. Rangelands 28(1):23-28.

Toevs, G.R., J.W. Karl, J.J. Taylor, C.S. Spurrier, M. Karl, M.R. Bobo, and J.E. Herrick. 2011 Consistent indicators and methods and a scalable sampling design to meet assessment, inventory, and monitoring information needs across scales. Rangelands 33(4):6-13.

Tongway, D.J., and N.L. Hindley. 2004. Landscape Function Analysis: Methods for monitoring and assessing landscapes, with special reference to minesites and rangelands. Canberra, Australia: CSIRO Sustainable Ecosystems. http://www.cse. csiro.au/research/efa/index.htm.
Trochim, W.M.K. 2006. Research Methods Knowledge Base. http://www.socialresearchmethods.net $/ \mathrm{kb} / \mathrm{scallik}$.php.

Walters, C. 2007. Is adaptive management helping to solve fisheries problems? Ambio 36:304-307.

Walters, C.J., and C.S. Holling. 1990. Large-scale management experiments and learning by doing. Ecology 71:2060-2068.

Washington-Allen, R.A., N.E. West, R.D. Ramsey, and R.E. Efroymson. 2006. A protocol for retrospective remote sensing-based ecological monitoring of rangelands. Rangeland Ecology and Management 59:19-29.

Wooton, E.O. 1908. The range problem in New Mexico. Agriculture Experiment Station Bulletin 66. Las Cruces, NM: New Mexico College of Agriculture and Mechanic Arts. 